\title{
Espiritualidade e resiliência na atenção domiciliar
}

\author{
Spirituality and resilience in home care
}

\section{Espiritualidad y resiliencia en la atención domiciliaria}

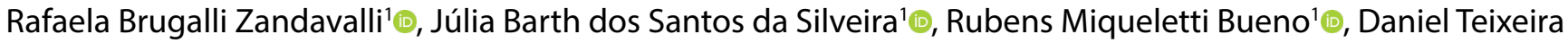 \\ dos Santos ${ }^{2} \odot$, Eno Dias de Castro Filho ${ }^{1} \odot$, Bruno Paz Mosqueiro ${ }^{1}(1)$ \\ ${ }^{1}$ Grupo Hospitalar Conceição, Porto Alegre, Rio Grande do Sul, Brasil \\ ${ }^{2}$ Hospital de Clínicas de Porto Alegre, Porto Alegre, Rio Grande do Sul, Brasil
}

\section{Resumo}

Introdução: A atenção domiciliar ( $A D)$ caracteriza-se por práticas de cuidado que realizam abordagem integral do paciente em seu contexto familiar, socioeconômico e cultural. Religiosidade e espiritualidade atuam como mecanismos de fortalecimento da resiliência. Objetivo: Avaliar a relação entre religiosidade e espiritualidade com resiliência em pacientes em $A D$ de Unidades de Saúde de Atenção Primária à Saúde (US-APS) de Porto Alegre (RS), Brasil. Métodos: estudo quantitativo transversal e descritivo, incluindo 44 adultos de quatro US-APS em $A D$ por condições crônicas e problemas de saúde controlados/compensados com alguma dependência para atividades da vida diária. Foram utilizadas escalas de religiosidade (DUREL), espiritualidade (ARES), resiliência (RS-14), funcionalidade (Katz), sintomas depressivos (PHQ2), suporte social (mMOS-SS), classificação econômica (ABEP 2016) e grau de severidade das condições clínicas (CIRS-G). Resultados: Pacientes avaliados são majoritariamente de sexo feminino $(72,7 \%)$, idosas (média 74 anos), viúvas, brancas, de baixo estrato socioeconômico, baixa escolaridade, aposentadas, com tempo médio de 7,5 anos de restrição domiciliar e grau moderado de resiliência. Os entrevistados apresentam altos índices de religiosidade e espiritualidade, sendo que $90,9 \%$ apresentam alta religiosidade intrínseca e $79,6 \%$ realizam práticas religiosas privadas uma ou mais vezes ao dia. A maioria $(88,6 \%)$ considera importante que sua religiosidade e espiritualidade seja abordada em seus atendimentos de saúde, mas somente $20,5 \%$ já foram questionados sobre tal temática. Resiliência associou-se à maior espiritualidade $(B=0,44 ; p=0,02)$, controlando-se este efeito para suporte social e sintomas depressivos; e à maior idade $(B=0,18 ; p=0,02)$. Conclusão: $O$ estudo corrobora a relevância da dimensão de religiosidade e espiritualidade e indica seu papel na promoção de resiliência nesta população em AD. Recomenda-se a abordagem da religiosidade e espiritualidade com esses indivíduos, fortalecendo o cuidado integral preconizado pela APS.

Descritores: Espiritualidade; Religião; Resiliência Psicológica; Assistência Domiciliar; Atenção Primária à Saúde.

Como citar: Zandavalli RB, Silveira JBS, Bueno RM, Santos DT, Castro Filho ED, Mosqueiro BP. Espiritualidade e resiliência na atenção domiciliar. Rev Bras Med Fam Comunidade. 2020;15(42):2213. https://doi.org/10.5712/rbmfc15(2)2213

\section{Autor correspondente:}

Rafaela Brugalli Zandavalli.

E-mail: rafaelazandavalli@gmail.com

Fonte de financiamento:

declaram não haver.

Parecer CEP:

17.317 (Grupo Hospitalar Conceição), aprovado em 19 de março de 2018.

Conflito de interesses:

declaram não haver.

Procedência e revisão por pares:

revisado por pares.

Recebido em: 27/09/2019

Aprovado em: 12/12/2019 


\begin{abstract}
Introduction: Home Care $(\mathrm{HC})$ is characterized by practices that perform integral approach of the patient in their family, socioeconomic and cultural context. Religiosity and spirituality as mechanisms for strengthening resilience. Objective: To evaluate the relationship between religiosity and spirituality with resilience in HC patients from Primary Health Care Units (PHC-U) of Porto Alegre (RS), Brazil. Methods: Cross-sectional and descriptive quantitative study including 44 adults from four $\mathrm{PHC}-\mathrm{U}$ in $\mathrm{HC}$ due to chronic conditions and controlled/compensated health problems with some dependence on activities of daily living. We used religiousness (DUREL), spirituality (ARES), resilience (RS-14), functionality (Katz), depressive symptoms (PHQ-2), social support (mMOS-SS), economic classification (ABEP 2016) and severity of clinical conditions (CIRS-G) scales. Results: Most of the evaluated patients were female ( $72.7 \%$ ), elderly (average 74 years), widows, white, low socioeconomic status, low education, retired, with an average time of 7.5 years of home restriction and with moderated degree of resilience. Respondents have high religiosity and spirituality rates, with $90.9 \%$ presenting high intrinsic religiosity and $79.6 \%$ performing private religious practices one or more times a day. Most ( $88.6 \%$ ) consider important that their religiosity and spirituality is addressed in their health care, but only $20.5 \%$ have been asked about it. Resilience was associated with higher spirituality $(B=0,44 ; p=0,02)$, controlling this effect for social support and depressive symptoms; and older age $(B=0,18 ; p=0,02)$. Conclusion: The study corroborates the relevance of the religiosity and spirituality dimension and indicates its role in promoting resilience in this population in $\mathrm{HC}$. The approach to religiosity and spirituality with these individuals is recommended, strengthening the integral care recommended by PHC.
\end{abstract}

Descriptors: Spirituality; Religion; Psychological Resilience; Home Care; Primary Health Care.

\title{
Resumen
}

Introducción: la atención domiciliaria $(A D)$ se caracteriza por prácticas de atención que realizan un enfoque integral del paciente en su contexto familiar, socioeconómico y cultural. La religiosidad y la espiritualidad actúan como mecanismos para fortalecer la resiliencia. Objetivo: evaluar la relación entre la religiosidad y la espiritualidad con resiliencia en pacientes en AD de las Unidades de Salud de la Atención Primaria a la Salud (US-APS) de Porto Alegre (RS), Brasil. Métodos: estudio cuantitativo descriptivo y transversal que incluyó a 44 adultos de cuatro US-APS en AD debido a afecciones crónicas y problemas de salud controlados/compensados con cierta dependencia de las actividades de la vida diaria. Utilizamos escalas de religiosidad (DUREL), espiritualidad (ARES), resiliencia (RS-14), funcionalidad (Katz), síntomas depresivos (PHQ-2), apoyo social (mMOS-SS), clasificación económica (ABEP 2016) y gravedad de las condiciones clínicas (CIRS - G). Resultados: la mayoría de los pacientes evaluados eran mujeres (72.7\%), ancianas (edad promedio 74 años), viudas, blancas, de bajo nivel socioeconómico, baja educación, jubiladas, con un tiempo promedio de 7.5 años de restricción domiciliaria y grado moderado de resiliencia. Los encuestados tienen altas tasas de la religiosidad y la espiritualidad, $90.9 \%$ tienen una alta religiosidad intrínseca y $79.6 \%$ realizan prácticas religiosas privadas una o más veces al día. La mayoría $(88,6 \%)$ considera importante que su la religiosidad y la espiritualidad se aborde en su atención médica, pero solo al $20,5 \%$ se les ha preguntado al respecto. La resiliencia se asoció con una mayor espiritualidad $(B=0,44 ; p=0,02)$, controlando este efecto para apoyo social y los síntomas depresivos, y la edad avanzada $(B=0,18 ; p=0,02)$. Conclusión: el estudio corrobora la relevancia de la dimensión religiosa y espiritual e indica su papel en la promoción de la resiliencia en esta población en AD. Se recomienda el enfoque de la religiosidad y la espiritualidad con estas personas, fortaleciendo la atención integral recomendada por la APS.

Descriptores: Espiritualidad; Religión; Resiliencia Psicológica; Atención Domiciliaria de Salud; Atención Primaria a la Salud.

\section{INTRODUÇÃO}

A atenção domiciliar (AD) é um conjunto de práticas de cuidado multiprofissional em domicílio que realiza abordagem integral do paciente em seu contexto familiar, socioeconômico e cultural. ${ }^{1}$ A maioria das condições associadas à necessidade de $A D$ são crônicas e causam significativo impacto na qualidade de vida. ${ }^{2} \mathrm{Na}$ América Latina, estima-se que a proporção da população acima de 60 anos aumentará de $12 \%$ em 2017 para $25 \%$ em 2050, ${ }^{3}$ sinalizando indiretamente uma demanda crescente de cuidados domiciliares.

Em contexto de inegáveis estressores, ${ }^{4}$ ser resiliente ou desenvolver resiliência é uma forma de superar os prejuízos ocasionados por diversas limitações presentes na população em AD. Resiliência é definida como a capacidade de adaptar-se de forma bem sucedida e recuperar-se após ter experienciado severa adversidade durante a vida. ${ }^{5}$ Políticas públicas visando cuidado à saúde mental são necessárias, uma vez que esta é um direito humano básico universal essencial e relevante para o desenvolvimento sustentável global, sendo a resiliência uma dimensão da saúde mental. ${ }^{6}$ Aspectos biológicos, psicológicos e sociológicos têm sido tradicionalmente relacionados como fatores determinantes de resiliência e o estudo recente da religiosidade e espiritualidade tem contribuído para o conhecimento dos caminhos que levam à resiliência. ${ }^{7}$ Identificar os fatores relacionados à resiliência pode auxiliar os profissionais de saúde a promovê-los. ${ }^{8}$ 
No Brasil, a maior parte da população considera a religião muito importante em sua vida, ${ }^{9}$ com destaque na relevância da religiosidade para indivíduos idosos, ${ }^{9,10}$ os quais compõem a maioria dos pacientes em AD. Religiosidade diz respeito ao quanto um indivíduo acredita, segue e pratica uma determinada religião. ${ }^{11}$ Espiritualidade é entendida como a busca pessoal de compreensão relacionada a questões existenciais maiores (por exemplo, o sentido da vida e a morte) e suas relações com o sagrado e/ou transcendente. Esse conceito de espiritualidade pode levar ao desenvolvimento de práticas religiosas ou na formação de comunidades religiosas, mas não necessariamente. ${ }^{11}$ Nas últimas décadas, evidências científicas vêm destacando a grande influência da religiosidade e espiritualidade na saúde física e mental, ${ }^{12}$ inclusive demonstrando impacto em mortalidade. ${ }^{13}$

O objetivo do atual estudo é avaliar a relação entre religiosidade e espiritualidade com resiliência em usuários em AD em Unidades de Atenção Primária à Saúde (APS) de Porto Alegre. Após revisão de literatura, percebe-se que este é o primeiro estudo a avaliar tal relação em pessoas em atendimento domiciliar.

\section{MÉTODOS}

\section{Desenho}

Trata-se de uma pesquisa quantitativa de caráter observacional e transversal.

\section{Local}

A pesquisa foi realizada nos territórios adscritos de quatro unidades de saúde da atenção primária à saúde (APS) pertencentes ao Serviço de Saúde Comunitária do Grupo Hospitalar Conceição, na cidade de Porto Alegre, Rio Grande do Sul, Brasil.

\section{Participantes}

Os participantes foram todos indivíduos maiores de 18 anos pertencentes à Atenção Domiciliar categoria $1(A D 1)^{14}$ das unidades de saúde SESC, Vila Floresta, Conceição e Barão de Bagé, moradores nos respectivos territórios durante o período de coleta que foram apontados pelas suas unidades em condições físicas e mentais de responder aos questionários aplicados. Estes pacientes recebem atendimento domiciliar por apresentarem problemas de saúde controlados/compensados e terem dificuldade ou impossibilidade física de locomoção até sua unidade de saúde. Foram excluídos os indivíduos que, no momento da entrevista, apresentaram dificuldades de compreender ou de responder aos questionários aplicados, pacientes que não conseguiram completar preenchimento dos questionários ou que residiam em regiões com alta periculosidade para acesso dos pesquisadores.

\section{Metodologia de coleta de dados}

Após serem referenciados pelas Unidades, as entrevistas foram combinadas individualmente por contato telefônico ou por intermédio das agentes comunitárias de saúde (ACS). As avaliações foram realizadas no domicílio dos entrevistados com instrumentos aplicados pelos pesquisadores (médicos residentes em Medicina de Família e Comunidade) através de leitura para o entrevistado. Foi permitido o auxílio de um familiar para responder às escalas de saúde física (CIRS), funcionalidade (Katz), classificação econômica (ABEP) e identificação. Para a escala CIRS, os prontuários dos pacientes também foram revisados. 


\section{Instrumentos}

\section{a) Dados demográficos e anamnese religiosa/espiritual}

O questionário de identificação é composto pelos seguintes itens: data de nascimento, sexo, cor, estado conjugal, anos de estudo, ocupação, vínculo atual com serviço de saúde, tempo de restrição domiciliar, religião e sincretismo religioso.

Questionamentos com respostas categóricas (sim/não) foram utilizados para avaliação de aspectos descritivos de religiosidade e espiritualidade: "Você acha importante os profissionais de saúde perguntarem sobre a religiosidade e espiritualidade nos atendimentos em saúde?”, "Algum profissional da área da saúde já perguntou sobre sua religiosidade ou espiritualidade em seus atendimentos em saúde?" e "Se sim, médico?".

Esta seção não possui perguntas com validação.

\section{b) Religiosidade}

A versão validada em português da escala Duke Religion Index (DUREL), ${ }^{15}$ também validada para a APS,${ }^{16}$ é uma escala Likert com 5 pontos, amplamente utilizada, que mede três dimensões de religiosidade. A primeira questão avalia religiosidade organizacional ( $R O$ - frequência a instituições religiosas como igrejas ou outros templos religiosos); a segunda avalia religiosidade não organizacional ou práticas religiosas privadas (atividades religiosas praticadas no privado como preces, leitura de textos religiosos e meditação) e as últimas três questões, religiosidade intrínseca, que compreende a busca pela vivência e incorporação das próprias crenças religiosas no modo próprio de vida, esforçando-se por internalizá-las e segui-las completamente. ${ }^{17} \mathrm{~A}$ religiosidade intrínseca pode ser usada como escore contínuo ou como divisão categórica. Religiosidade intrínseca alta foi definida como um escore geral nas três questões maior que 10 pontos. ${ }^{18}$

\section{c) Espiritualidade}

A espiritualidade foi avaliada através da Escala de Atitudes Relacionadas à Espiritualidade (ARES), que foi a primeira escala desenvolvida e validada no contexto brasileiro, de acordo com os autores. Consiste de 11 perguntas respondidas em escala Likert de 5 pontos cuja soma produz um escore no qual quanto maior o número, maior o envolvimento do indivíduo com espiritualidade e com práticas referentes à orientação espiritual. Ela avalia 4 dimensões: crenças espirituais, práticas espirituais, experiências espirituais e atitudes/consequências. ${ }^{19}$

\section{d) Resiliência}

Utilizou-se a Escala de Resiliência (RS-14) validada para o português. ${ }^{20} \mathrm{O}$ instrumento consiste de 14 itens com uma escala Likert de 7 pontos e tem 5 domínios: perseverança, autoconfiança, serenidade, sentido de vida e autossuficiência. ${ }^{20}$ A medida foi analisada como escore global, de forma que maiores escores na RS-14 indicam maior resiliência (variação de 7 a 98 pontos). De acordo com o escore global, pode-se classificar os níveis de resiliência em muito baixos, baixos, no limite inferior, moderados, moderadamente altos e altos. ${ }^{21}$ 


\section{e) Outros instrumentos}

Outros instrumentos foram utilizados para avaliação de características clínicas e sociodemográficas relevantes para o entendimento da população e sua relação com religiosidade e resiliência.

A Escala de Katz ou Escala de Independência em Atividades da Vida Diária (EIAVD), ${ }^{22}$ validada para o Brasil,, ${ }^{23}$ que é composta por seis itens (tomar banho, vestir-se, uso do vaso sanitário, transferência, continência e alimentação) foi utilizada para avaliação da funcionalidade dos usuários. Para os resultados, os pacientes foram agrupados em 3 grupos conforme seu nível de funcionalidade, baseado na padronização proposta pelo Hartford Institute for Geriatric Nursing. ${ }^{24}$

A avaliação de sintomas depressivos foi avaliada com o instrumento de rastreamento para depressão Patient Health Questionnaire - two items (PHQ-2), validado para o Brasil. ${ }^{25}$

Para avaliação do suporte social, foi utilizado o questionário eight-item modified Medical Outcomes Study Social Support Survey (eight-item mMOS-SS), ${ }^{26}$ utilizando como base o questionário original Medical Outcomes Study (MOS) ${ }^{27}$ validado para o português brasileiro. ${ }^{28}$

A classificação econômica foi avaliada com o questionário da Associação Brasileira de Empresas de Pesquisa (ABEP) - 2016. ${ }^{29}$

A escala Cumulative Illness Rating Scale - Geriatric (CIRS-G), ${ }^{30}$ não validada para o português, porém validada para o contexto da atenção primária à saúde (APS),${ }^{31}$ foi utilizada para avaliar o grau de severidade das condições clínicas.

\section{Análises estatísticas}

Aspectos descritivos relacionados à importância da religiosidade e espiritualidade na população em $A D$ foram avaliados através de frequências absolutas e relativas das variáveis investigadas e descritas. Somente foram incluídos para análise os sujeitos que completaram a integridade dos instrumentos. Foram realizadas análises univariadas através de regressões lineares para investigar a associação entre variáveis clínicas estudadas e com resiliência. Diante de tamanho amostral final menor e menor poder estatístico em relação ao cálculo amostral prévio de 70 indivíduos, que considerava um poder de $80 \%$ e nível de significância de $5 \%$ tendo como base o estudo por Mosqueiro, ${ }^{32}$ optado por diversos modelos multivariáveis contendo resiliência (variável dependente), espiritualidade e outras variáveis independentes estatisticamente significativas $(p<0,05)$ ou com tendência a esta significância $(p<0,25)$.

\section{Aspectos éticos}

Este estudo foi aprovado pelo Comitê de Ética em Pesquisa do Grupo Hospitalar Conceição sob o parecer de $n^{\circ} 17317$ e todos os pacientes incluídos preencheram o Termo de Consentimento livre e Esclarecido antes de entrar no estudo. Não houve participação de pacientes no planejamento da pesquisa. Os dados serão disponibilizados a outros pesquisadores mediante projeto de pesquisa com aprovação ética. 


\section{RESULTADOS}

De 60 pacientes identificados entre março e agosto de 2018, 44 foram incluídos no estudo (Figura 1).

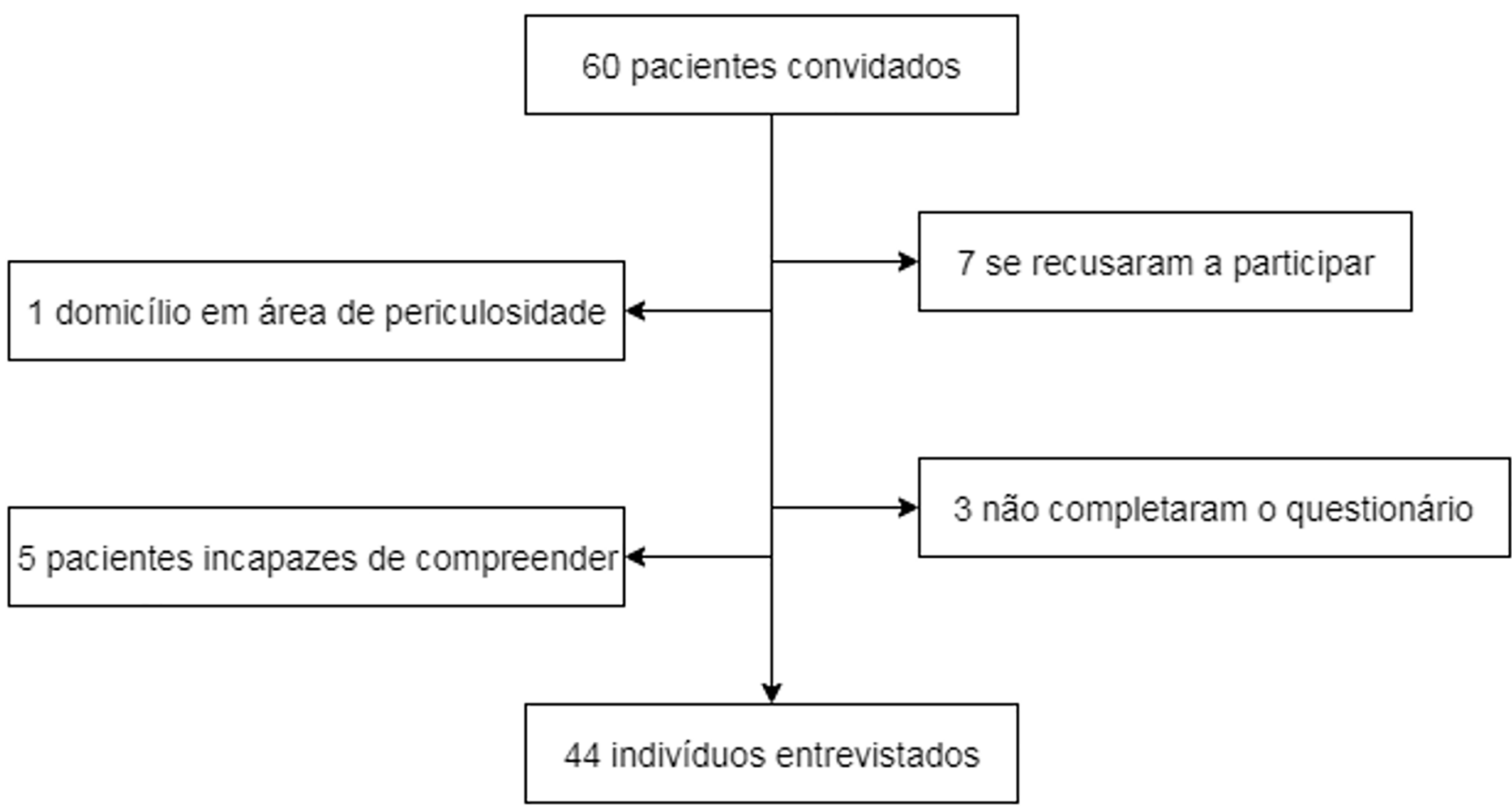

Figura 1. Fluxograma do processo de inclusão dos pacientes.

As características socioeconômicas e clínicas da população estudada estão detalhadas na Tabela 1. A população estudada caracterizou-se majoritariamente como idosa, feminina, branca, viúva, de baixo estrato socioeconômico, baixa escolaridade e aposentada. Aproximadamente um terço apresentou rastreio positivo para depressão; quase metade foi classificada como independente para as atividades de vida diária.

As condições mais graves que podem justificar a restrição ao domicílio pertencem aos sistemas (pontuação 3 ou 4 na escala CIRS-G): músculo esquelético/tegumento (61,3\%); neurológico (27,3\%); respiratório $(6,8 \%)$; endócrino/metabólico e mama $(6,8 \%)$; coração $(4,5 \%)$; vascular; hematopoiético; otorrinolaringológico/oftalmológico; trato gastrointestinal superior; fígado/pancreático e doença psiquiátrica $(2,3 \%$ cada um).

As comorbidades presentes nos participantes foram as seguintes: HAS (77,3\%); DM (31,8\%); osteoartrite (29,5\%); sequela de AVC (25\%); depressão (20,5\%); incontinência urinária (18,2\%); DPOC $(13,6 \%)$; sequela de fratura/cirurgia $(13,6 \%)$; oftalmopatias $(11,4 \%)$; neoplasia $(9,1 \%)$; cardiopatia Isquêmica $(9,1 \%)$; distúrbios do ritmo cardíaco $(9,1 \%)$; doenças da tireoide $(6,8 \%)$; insuficiência cardíaca $(6,8 \%)$; amputação de membros; paralisia infantil; valvopatias; Parkinson; HIV; obesidade mórbida (4,5\% cada um) e aneurisma $(2,3 \%)$. 
Tabela 1. Características socioeconômicas e clínicas da amostra $(n=44)$.

\begin{tabular}{|c|c|c|c|c|c|}
\hline \multicolumn{2}{|l|}{ Variável } & Média & $\mathrm{DP}^{*}$ & Mín & Máx \\
\hline \multicolumn{2}{|l|}{ Idade } & 73,95 & 2,56 & 35 & 97 \\
\hline \multicolumn{2}{|l|}{ Anos de Estudo } & 6,3 & 0,53 & 0 & 14 \\
\hline \multicolumn{2}{|l|}{ Anos de Restrição Domiciliar } & 7,48 & 1,21 & 0,16 & 41 \\
\hline \multicolumn{2}{|l|}{ Resiliência (RS-14) } & 78,18 & 1,36 & 51 & 98 \\
\hline \multicolumn{2}{|c|}{ Grau de Severidade de Condições Clínicas (CIRS) } & 6,86 & 1,9 & 3 & 13 \\
\hline \multicolumn{2}{|l|}{ Suporte Social (mMOS-SS) } & 35,56 & 0,93 & 15 & 40 \\
\hline & & \multicolumn{2}{|c|}{$\mathbf{N}$} & \multicolumn{2}{|c|}{$\%$} \\
\hline Sexo & Feminino & \multicolumn{2}{|c|}{32} & \multicolumn{2}{|c|}{72,7} \\
\hline \multirow[t]{2}{*}{ Etnia - Cor } & Branco & \multicolumn{2}{|c|}{34} & \multicolumn{2}{|c|}{77,3} \\
\hline & Pardo/Preto & \multicolumn{2}{|c|}{10} & \multicolumn{2}{|c|}{22,7} \\
\hline \multirow[t]{4}{*}{ Estado Conjugal } & Nunca teve companheiro(a) & \multicolumn{2}{|c|}{7} & \multicolumn{2}{|c|}{15,9} \\
\hline & Com companheiro(a) & \multicolumn{2}{|c|}{15} & \multicolumn{2}{|c|}{34,1} \\
\hline & Separado(a) & \multicolumn{2}{|c|}{2} & \multicolumn{2}{|c|}{4,5} \\
\hline & Viúvo(a) & \multicolumn{2}{|c|}{20} & \multicolumn{2}{|c|}{45,5} \\
\hline \multirow[t]{4}{*}{ Ocupação } & Dolar & \multicolumn{2}{|c|}{1} & \multicolumn{2}{|c|}{2,3} \\
\hline & Aposentado - tempo de serviço & \multicolumn{2}{|c|}{20} & \multicolumn{2}{|c|}{45,5} \\
\hline & Em auxílio-doença & & & & \\
\hline & Outro & & & & \\
\hline Vínculo com Serviço de Saúde & US SESC & & & & \\
\hline & US Vila Floresta & & & & \\
\hline & US Barão de Bagé & & & & \\
\hline & US Conceição & & & & \\
\hline Classe Social (ABEP) & B2 - renda média domiciliar $\mathrm{R} \$ 4.852$ & & & & \\
\hline & $\mathrm{C} 1$ - renda média domiciliar $\mathrm{R} \$ 2.705$ & & & & \\
\hline & $\mathrm{C} 2$ - renda média domiciliar $\mathrm{R} \$ 1.625$ & & & & \\
\hline & $\mathrm{D}-\mathrm{E}$ - renda média domiciliar $\mathrm{R} \$ 768$ & & & & \\
\hline PHQ-2 & Rastreio + para Depressão & & & & \\
\hline & Rastreio - para Depressão & & & & \\
\hline Funcionalidade (Katz) & Independente & & & & \\
\hline & Dependência Moderada & & & & \\
\hline & Muito Dependente & & & & \\
\hline
\end{tabular}

${ }^{*} \mathrm{DP}=$ desvio padrão

As características de religiosidade estão descritas na Tabela 2. A maioria dos pacientes se mostrou favorável ao questionamento sobre sua religiosidade e espiritualidade por profissionais de saúde durante atendimentos. No entanto, apenas $20 \%$ relataram já terem sido questionados sobre o tema. A RI e a frequência de práticas religiosas privadas foram elevadas, ao passo que o comparecimento a templos religiosos foi baixo.

A avaliação da espiritualidade segundo a escala ARES está presente na Tabela 3.

Os resultados da análise univariada entre resiliência e as demais variáveis investigadas neste estudo estão descritas na Tabela 4. Houve correlação estatisticamente significativa entre resiliência e espiritualidade (Figura 2), além de resiliência e idade (Figura 3). 
Tabela 2. Religiosidade.

\begin{tabular}{|c|c|c|c|}
\hline & & $\mathbf{N}$ & $\%$ \\
\hline \multirow{2}{*}{$\begin{array}{l}\text { Você acha importante os profissionais de saúde perguntarem sobre a religiosidade } \\
\text { e espiritualidade nos atendimentos em saúde? }\end{array}$} & Sim & 39 & 88,6 \\
\hline & Não & 5 & 11,4 \\
\hline \multirow{2}{*}{$\begin{array}{l}\text { Algum profissional da área da saúde já perguntou sobre sua religiosidade ou } \\
\text { espiritualidade em seus atendimentos em saúde? }\end{array}$} & Sim & 9 & 20,5 \\
\hline & Não & 35 & 79,5 \\
\hline \multirow{2}{*}{ Se sim, médico? } & Sim & 7 & 77,8 \\
\hline & Não & 2 & 22,2 \\
\hline \multirow{6}{*}{ Você possui alguma denominação religiosa? } & Católico & 28 & 63,6 \\
\hline & Evangélico & 3 & 6,8 \\
\hline & Afro-brasileiras & 3 & 6,8 \\
\hline & Espírita & 2 & 4,5 \\
\hline & Sem religião, com espiritualidade & 7 & 15,9 \\
\hline & Outro & 1 & 2,3 \\
\hline \multirow{2}{*}{ Segue/pratica mais de uma religião? } & Sim & 15 & 34,1 \\
\hline & Não & 29 & 65,9 \\
\hline \multirow{4}{*}{ Se sim, qual? } & Evangélico+Católico & 4 & 26,7 \\
\hline & Umbanda+Católico & 4 & 26,7 \\
\hline & Espírita+Católico & 2 & 13,3 \\
\hline & Outros & 5 & 33,3 \\
\hline \multirow{2}{*}{ Religiosidade Intrínseca (DUREL) } & Alta & 40 & 90,9 \\
\hline & Baixa & 4 & 9,1 \\
\hline \multirow{3}{*}{ Frequência a templo religioso (DUREL) } & 1 ou + vezes/semana & 5 & 11,3 \\
\hline & Algumas vezes/ano & 7 & 15,9 \\
\hline & 1 vez/ano ou nunca & 32 & 72,8 \\
\hline \multirow{3}{*}{ Práticas Religiosas Privadas - preces, leitura textos religiosos, etc. (DUREL) } & 1 ou + vezes/dia & 35 & 79,6 \\
\hline & 1 ou + vezes/semana & 7 & 15,9 \\
\hline & Raramente ou nunca & 2 & 4,5 \\
\hline
\end{tabular}

Tabela 3. Espiritualidade - Questionário ARES.

\begin{tabular}{|c|c|c|c|c|c|}
\hline & \multicolumn{5}{|c|}{$\mathbf{N}(\%)$} \\
\hline & $\begin{array}{l}\text { Discordo } \\
\text { Muito }\end{array}$ & $\begin{array}{l}\text { Discordo } \\
\text { Parcialmente }\end{array}$ & $\begin{array}{l}\text { Não concordo } \\
\text { nem discordo }\end{array}$ & $\begin{array}{l}\text { Concordo } \\
\text { parcialmente }\end{array}$ & $\begin{array}{c}\text { Concordo } \\
\text { muito }\end{array}$ \\
\hline $\begin{array}{l}\text { Eu acredito em algo sagrado, transcendente (Deus, uma força } \\
\text { superior). }\end{array}$ & - & - & - & $3(6,8)$ & $41(93,2)$ \\
\hline $\begin{array}{l}\text { Meditação, oração, leituras e/ou contemplação são práticas } \\
\text { que utilizo (ao menos uma delas) para me conectar com uma } \\
\text { força espiritual além de mim. }\end{array}$ & $2(4,5)$ & $1(2,3)$ & - & $7(15,9)$ & $34(77,3)$ \\
\hline $\begin{array}{l}\text { Já presenciei fatos/situações que me levaram a acreditar que } \\
\text { existe algo além do mundo material. }\end{array}$ & $8(18,2)$ & $3(6,8)$ & $1(2,3)$ & $8(18,2)$ & $24(54,5)$ \\
\hline Minha fé ou crenças espirituais me dão apoio no dia a dia. & $1(2,3)$ & $1(2,3)$ & - & $8(18,2)$ & $34(77,3)$ \\
\hline $\begin{array}{l}\text { Minha espiritualidade me ajuda a ter um relacionamento } \\
\text { melhor com os outros. }\end{array}$ & $3(6,8)$ & $1(2,3)$ & $1(2,3)$ & $11(25,0)$ & $28(63,6)$ \\
\hline Minha espiritualidade influencia minha saúde física e mental. & $2(4,5)$ & - & $2(4,5)$ & $5(11,4)$ & $35(79,5)$ \\
\hline Minha espiritualidade me incentiva a ajudar outras pessoas. & $2(4,5)$ & - & $1(2,3)$ & $5(11,4)$ & $36(81,8)$ \\
\hline Eu acredito em uma continuidade após a morte. & $6(13,6)$ & $2(4,5)$ & $7(15,9)$ & $6(13,6)$ & $23(52,3)$ \\
\hline $\begin{array}{l}\text { Minhas crenças e valores espirituais direcionam minhas ações } \\
\text { no dia a dia. }\end{array}$ & $3(6,8)$ & $2(4,5)$ & $2(4,5)$ & $9(20,5)$ & $28(63,6)$ \\
\hline Minha fé ou crenças espirituais dão sentido à minha vida. & $2(4,5)$ & - & $1(2,3)$ & $4(9,0)$ & $37(84,0)$ \\
\hline \multirow{2}{*}{$\begin{array}{l}\text { Práticas espirituais (por exemplo: fazer orações, ou jejum, ou } \\
\text { meditação ou outras) ajudam a manter ou melhorar a minha } \\
\text { saúde física ou mental. }\end{array}$} & $2(4,5)$ & $1(2,3)$ & - & $5(11,4)$ & $36(81,8)$ \\
\hline & & Média & DP* & Mín & Máx \\
\hline Espiritualidade (ARES) & & 49,13 & 1,11 & 23 & 55 \\
\hline
\end{tabular}


Tabela 4. Correlações entre resiliência e variáveis biopsicossociais.

\begin{tabular}{|c|c|c|c|}
\hline Variáveis & B (IC 95\%) & $\mathbf{R}^{2}$ & p \\
\hline Espiritualidade (ARES) & $0,44(0,08$ a 0,79$)$ & 0,13 & $0,02^{*}$ \\
\hline Religiosidade Intrínseca (DUREL) & $0,25(-0,77$ a 1,28$)$ & 0,01 & 0,62 \\
\hline Práticas Religiosas Privadas (DUREL) & $1,35(-0,85$ a 3,55$)$ & 0,04 & 0,22 \\
\hline Depressão (PHQ-2) & $-0,89(-2,33$ a 0,55$)$ & 0,04 & 0,22 \\
\hline Suporte Social (MOS) & $0,23(-0,15$ a 0,73$)$ & 0,04 & 0,20 \\
\hline Funcionalidade (Katz) & $-0,37(-1,89$ a 1,14$)$ & 0,01 & 0,62 \\
\hline Idade & $0,18(0,03$ a 0,34$)$ & 0,12 & $0,02^{*}$ \\
\hline Sexo & $-0,21(-6,45$ a 6,04$)$ & 0 & 0,95 \\
\hline Cor - Etnia & $0,28(-6,35$ a 6,92$)$ & 0 & 0,93 \\
\hline Estado Conjugal (com companheiro x nunca teve companheiro, viúvo ou separado) & $-2,46(-8,27$ a 3,36$)$ & 0,02 & 0,40 \\
\hline Anos de Estudo & $0,42(-0,37$ a 1,22$)$ & 0,03 & 0,29 \\
\hline Anos de Restrição Domiciliar & $-0,19(-0,54$ a 0,15$)$ & 0,03 & 0,26 \\
\hline Grau de Severidade de Condições Clínicas (CIRS) & $0,48(-0,10$ a 1,95$)$ & 0,01 & 0,52 \\
\hline
\end{tabular}

* Significância estatística $(p<0,05)$; As variáveis Religiosidade Intrínseca e Práticas Religiosas Privadas pontuam menos quanto maiores elas são, motivo pelo qual foram analisadas com valores invertidos; $\mathrm{B}=\mathrm{o}$ quanto aumentará a variável dependente (resiliência) a cada aumento em 1 unidade na variável independente. Apresenta semelhança com o conceito da variável " $r$ " utilizado em correlações de Pearson; $R^{2}=$ valor de 0 a 1 que pode ser entendido como porcentagem que estima o quanto a variável independente explica a diferença na variável dependente. Exemplo: $R^{2}$ de $0,1=10 \%$.

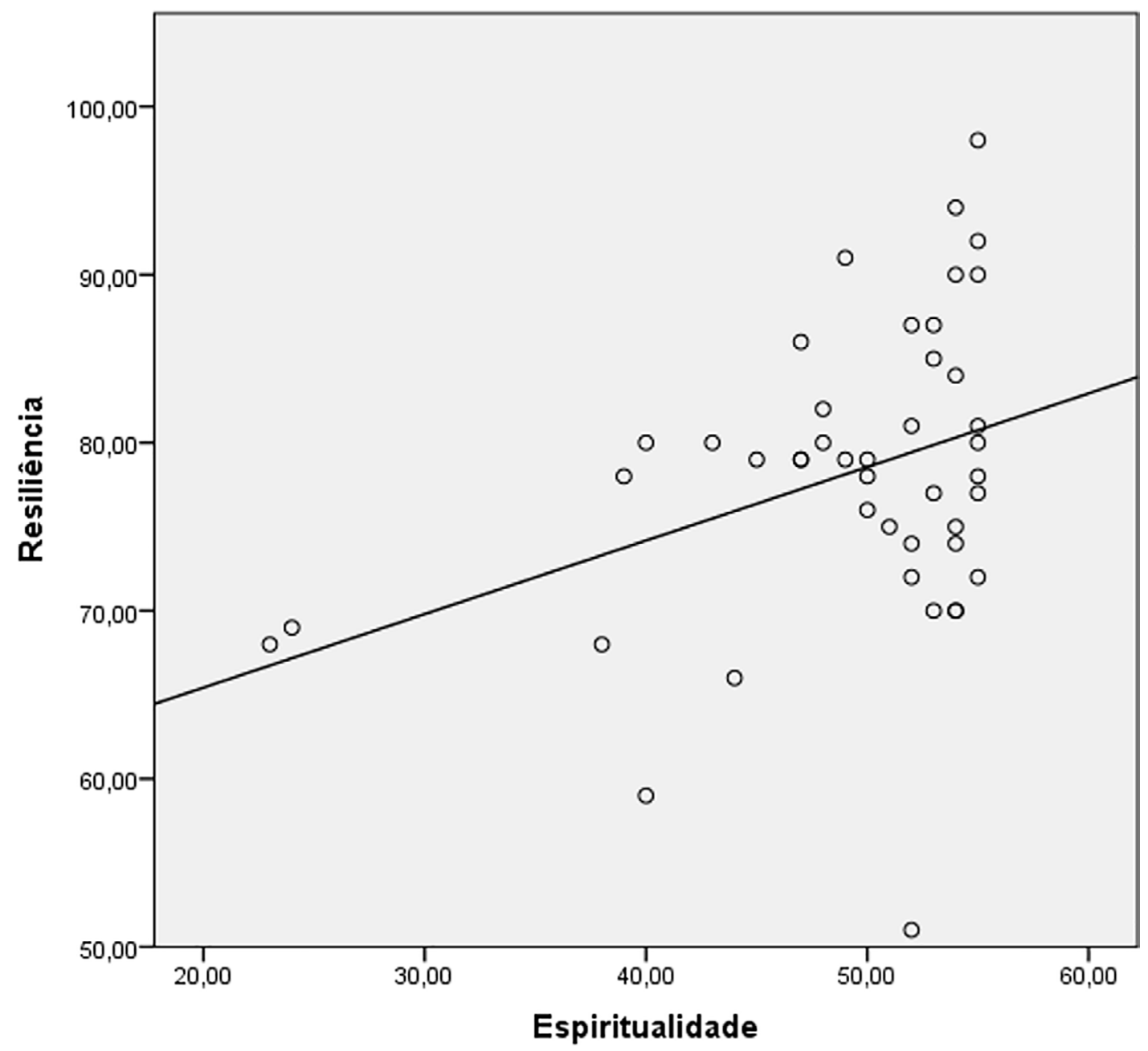

Figura 2. Gráfico de correlação entre resiliência e espiritualidade $(p=0,02 ; B=0,44)$. 


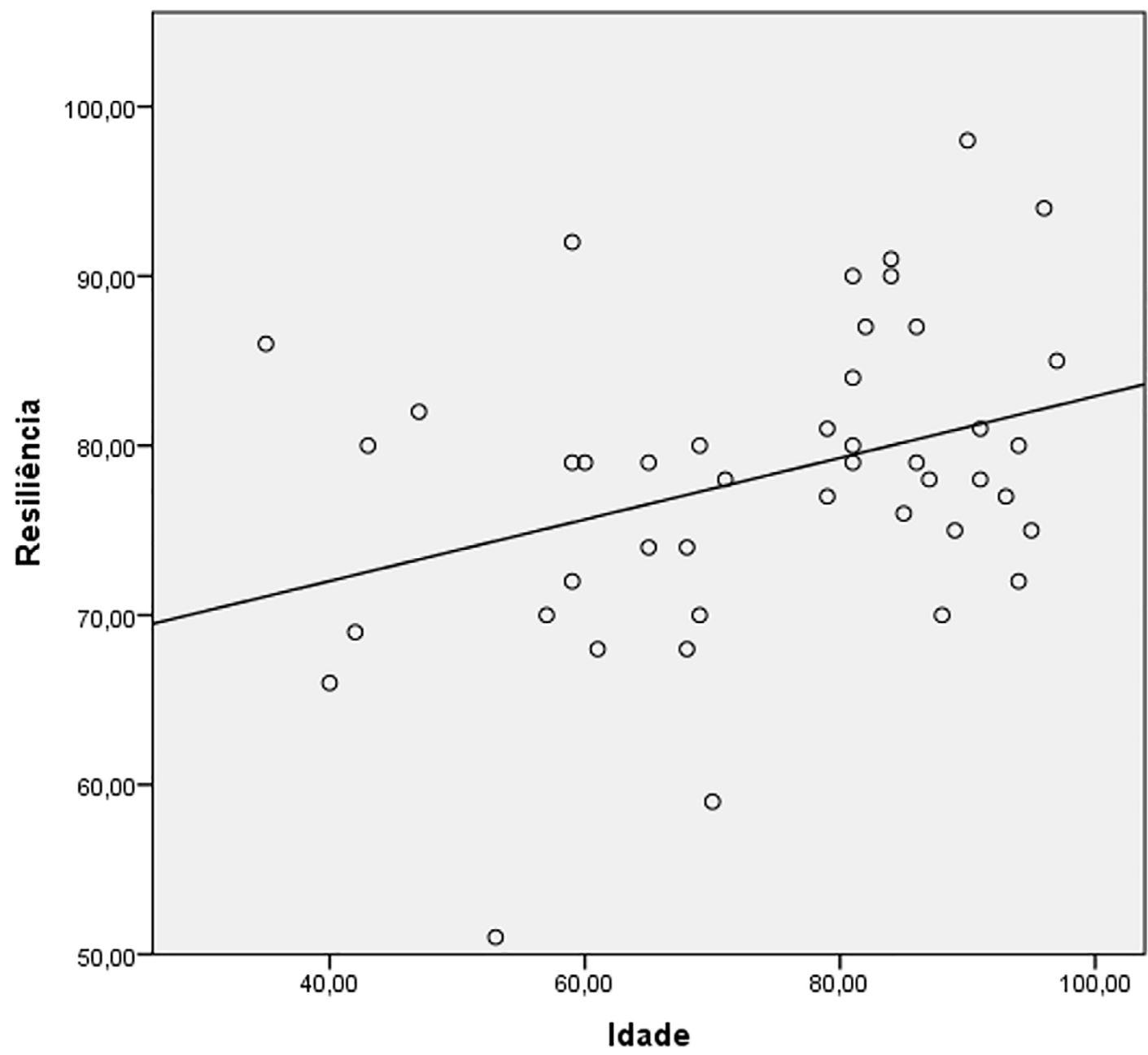

Figura 3. Gráfico de correlação entre resiliência e idade $(p=0,02 ; B=0,18)$.

Análises de regressão linear identificaram uma relação estatisticamente significativa entre espiritualidade e resiliência em modelos com significância estatística controlados para depressão e suporte social (Tabela 5). Outra análise realizada demonstrou significância estatística para espiritualidade $(p=0,042)$ em um modelo com tendência à significância $(p=0,08)$ utilizando a variável dependente como resiliência e independentes como espiritualidade, sintomas depressivos $(p=0,543)$ e suporte social $(p=0,468)$. Quando se inclui a idade como outra variável a este modelo, ele fica com significância geral de 0,043 e os valores $p$ de cada variável independente são: idade $(p=0,08)$, espiritualidade $(p=0,299)$, suporte social $(p=0,269)$ e sintomas depressivos $(p=0,442)$. 
Tabela 5. Modelos multivariáveis de resiliência como variável dependente.

\begin{tabular}{|c|c|c|}
\hline & B (IC 95\%) & $p$ \\
\hline \multicolumn{3}{|l|}{ Modelo $1^{*}$} \\
\hline Espiritualidade & $0,32(-0,07$ a 0,71$)$ & 0,11 \\
\hline Idade & $0,12(-0,05$ a 0,29$)$ & 0,15 \\
\hline \multicolumn{3}{|l|}{ Modelo $2^{*}$} \\
\hline Espiritualidade & $0,41(0,04$ a 0,77$)$ & $0,03^{*}$ \\
\hline Suporte Social & $0,19(-0,24$ a 0,62$)$ & 0,37 \\
\hline \multicolumn{3}{|l|}{ Modelo $3^{*}$} \\
\hline Espiritualidade & $0,41(0,04$ a 0,77$)$ & $0,03^{*}$ \\
\hline Depressão & $-0,56(-2,0$ a 0,84$)$ & 0,42 \\
\hline \multicolumn{3}{|l|}{ Modelo 4} \\
\hline Espiritualidade & $0,46(0,02$ a 0,90$)$ & $0,04^{*}$ \\
\hline Práticas Religiosas Privadas & $0,24(-2,37$ a 2,85$)$ & 0,85 \\
\hline
\end{tabular}

* Significância estatística $(p<0,05)$; B: o quanto aumentará a variável dependente (resiliência) a cada aumento em 1 unidade na variável independente. Apresenta semelhança com o conceito da variável " $r$ " utilizado em correlações de Pearson; $R^{2}=$ valor de 0 a 1 que pode ser entendido como porcentagem que estima o quanto a variável independente explica a diferença na variável dependente. Exemplo: $R^{2}$ de $0,1=10 \%$; A variável Práticas Religiosas Privadas pontua menos quanto maior elas é, motivo pelo qual foi analisada com valores invertidos. Modelo 1 : Sig 0,02 . $R^{2}=0,17$. $R^{2} A j u s t a d o=0,13$. Tolerância de Colinearidade =0,81. VIF 1,23; Modelo 2: Sig 0,04. $R^{2}=0,14 \cdot R^{2}$ Ajustado $=0,15$. Tolerância de Colinearidade $=0,96$. VIF 1,04; Modelo 3: Sig 0,04. $R^{2}=0,14$. $R^{2}$ Ajustado $=0,1$. Tolerância de Colinearidade $=0,96$. VIF 1,05; Modelo 4: Sig 0,06. $R^{2}=0,13 . R^{2}$ Ajustado $=0,09$. Tolerância de Colinearidade $=0,66$. VIF 1,52

\section{DISCUSSÃO}

\section{Resumo dos principais achados}

A população estudada apresentou elevados índices de vivência, valorização e interesse em atividades religiosas e/ou espirituais. A religiosidade no âmbito individual e privativo foi o principal mecanismo de manifestação da espiritualidade, e alguns ainda permaneciam frequentando o templo religioso regularmente, mesmo com limitações físicas. Houve evidente interesse por parte dos entrevistados quanto à abordagem da religiosidade e espiritualidade durante seus atendimentos de saúde, a despeito do seu escasso questionamento. A média na escala global de resiliência caracteriza a população estudada como tendo um grau moderado de resiliência. ${ }^{21}$

Observou-se que resiliência apresentou relação estatisticamente significativa com espiritualidade e idade, ambas explicando, respectivamente, 13\% e 12\% da variação observada em resiliência nas análises univariadas. A cada elevação de 1 ponto em escalas de espiritualidade e na idade, ocorria um aumento de, respectivamente, 0,44 (IC 95\% 0,08 a 0,79) e 0,18 (IC 95\% 0,03 a 0,34) na pontuação de resiliência. Embora significativo, o modelo multivariável envolvendo resiliência, espiritualidade e idade resultou em perda de significância estatística de espiritualidade e idade individualmente (possivelmente por conta de correlação entre essas variáveis), tendo também não contribuído para melhor explicar resiliência em relação aos modelos univariados (explicou, em conjunto, $13 \%$ da influência em resiliência). As variáveis depressão, suporte social e práticas religiosas privadas (com tendência à significância estatística na análise univariada), quando incluídas em modelos multivariáveis, não foram significativas e não provocaram contribuição para explicar resiliência. Nestes modelos, espiritualidade manteve-se significativa quando controlada para depressão e suporte social. 


\section{Fortalezas e limitações}

Por ser uma população de baixo nível socioeconômico, de faixa etária avançada e com comorbidades, houve limitações relacionadas à interpretação dos instrumentos, caracterizando um possível importante viés. Visando controlar esses fatores, os instrumentos foram lidos para os indivíduos pelos pesquisadores e os pacientes cujo entendimento foi insuficiente foram excluídos. Futuros estudos voltados a essa população devem levar em conta a capacidade cognitiva e nível educacional dos participantes. Uma outra possível limitação a considerar seria a ausência de um acesso direto dos pesquisadores à seleção da população em AD estudada, uma vez que foram avaliados consecutivamente somente os indivíduos referenciados pelas Unidades de Saúde, cada uma com formas de organização e monitorização própria destas populações. Também pode-se analisar que a quantidade de sujeitos analisada pode comprometer a validade externa do estudo.

O estudo é original, e, após revisão de literatura, constata-se que é o primeiro em nosso conhecimento a avaliar a religiosidade e a espiritualidade em uma população em AD brasileira. Estudou de forma quantitativa aspectos que, com frequência, são estudados qualitativamente. Foram utilizadas escalas validadas e a primeira escala de espiritualidade desenvolvida no Brasil (ARES). Toda a população em AD referenciada pelas quatro unidades de saúde foi avaliada pelos pesquisadores. Análise multivariada foi realizada visando controlar os fatores de confusão para a associação entre espiritualidade e resiliência.

Este é um estudo transversal que não permite realizar inferências causais. Os achados são iniciais e necessitam ser replicados e aprofundados, possivelmente avaliando outras e maiores populações em atendimento domiciliar, com diferentes níveis socioeconômicos e de outras regiões do Brasil.

\section{Comparação com a literatura}

O Brasil está entre os 10 países mais religiosos do mundo. ${ }^{10}$ Em um estudo com uma amostra probabilística da população brasileira ( $n=3.007$ ), a maior parte $(95 \%)$ indica uma filiação religiosa, $90 \%$ frequenta igrejas ou templos religiosos, $83 \%$ considera a religião muito importante em sua vida e $37 \%$ frequentam serviços religiosos pelo menos uma vez por semana. As filiações religiosas mais frequentes no Brasil são: Católica (68\%), Protestante/ Evangélica (23\%) e Espiritismo Kardecista (2,5\%). Pouco mais de 10\% das pessoas relataram frequentar mais de uma religião, ${ }^{9}$ aspecto característico da população brasileira. No presente estudo, a maioria dos pacientes em $A D$ também tinha níveis elevados de religiosidade e espiritualidade e era predominantemente católica; contudo, provavelmente devido à limitação física, a frequência a serviços religiosos era muito menor. Destacase também a elevada prevalência de sincretismo religioso em nossos pacientes $(34,1 \%)$ em contraposição ao dado apontado na literatura (10\%), o que sugere a existência de um padrão distinto em indivíduos em AD em relação à população geral ou a características deste território, ou se deve a uma forma diferente de avaliação em relação a outros estudos (seguir/praticar versus frequentar outra religião, respectivamente).

No levantamento epidemiológico representativo da população brasileira, maior idade e sexo feminino se associam à importância da religião e à maior frequência a serviços religiosos, mesmo após o controle para outras variáveis. ${ }^{9}$ Dados indicam que residir na Região Sul do Brasil e estado civil solteiro estão associados a menor importância da religião. ${ }^{9}$ Nesta pesquisa, embora todos os participantes sejam da região Sul do Brasil, a amostra analisada teve perfil predominantemente feminino, idoso e com histórico conjugal atual ou prévio, achados que podem se relacionar aos elevados índices de religiosidade e espiritualidade encontrados no estudo. 
Outro aspecto a destacar é a concordância majoritária com a maior parte das perguntas presentes na escala de espiritualidade. Nas questões sobre crenças em uma continuidade após a morte e sobre se o entrevistado já havia presenciado fatos/situações que lhe levaram a acreditar que exista algo além do mundo material, apesar de a maior parte concordar, estes foram os aspectos menos aceitos em comparação aos demais. A escala ARES foi validada para uma população com maior escolaridade, portanto algumas questões podem não ter sido bem compreendidas e interpretadas pela nossa população, o que pode explicar este achado.

Quanto ao desejo dos pacientes pela abordagem da religiosidade e espiritualidade em atendimentos de saúde, no Brasil, um estudo com 110 pacientes acima de 60 anos que frequentavam um serviço de reabilitação demonstrou que $69,5 \%$ consideraram como muito importante o impacto da sua fé e crenças em sua reabilitação e que $87,3 \%$ gostariam que seu médico lhe questionasse sobre sua fé e religião como parte de seu cuidado médico. Porém, os profissionais que assistiram esses pacientes somente haviam feito tal questionamento a $8,2 \%$ destes. ${ }^{33}$ Um estudo com 921 pacientes majoritariamente de clínicas de saúde da família norte-americanas encontrou que $83 \%$ gostaria que os médicos lhes questionassem sobre suas crenças espirituais pelo menos em algumas circunstâncias, sendo o desejo de entendimento entre médico e paciente o motivo mais importante. ${ }^{34} \mathrm{Um}$ estudo em pacientes de ambulatório de pneumologia demonstrou que $94 \%$ dos pacientes com crenças religiosas e $45 \%$ dos pacientes que negaram ter crenças religiosas gostariam de ser questionados sobre questões espirituais se estivessem gravemente doentes. ${ }^{35}$

Sobre esse tema, nossos resultados estão de acordo com a literatura e reforçam a existência de uma lacuna entre o desejo dos pacientes quanto a abordagem de seus aspectos religiosos e/ou espirituais durante uma consulta e a falta de questionamento sobre o tema, problema que se apresenta em diversos contextos do cuidado em saúde. Tal achado reforça a importância do reconhecimento e treinamento para abordagem da espiritualidade na prática clínica por profissionais da APS. Estudo com 292 Médicos de Família e Comunidade (MFC) brasileiros mostrou que, embora a grande maioria acredite que a religiosidade e a espiritualidade influencie muito a saúde $(88,4 \%)$ e que essa abordagem seja pertinente à prática clínica do MFC $(81,2 \%)$, apenas $35,0 \%$ da amostra se sentia preparada para abordar o tema e a maioria o faz com frequência moderada $(50,3 \%)$. As principais barreiras para essa abordagem seriam falta de tempo $(53,4 \%)$, treinamento $(39,7 \%)$ e valores pessoais, como medo de impor sua religião $(27,7 \%)$ ou medo de ofender o paciente $(23,6 \%)^{36}$, que poderiam ser superadas com o estudo e treinamento adequado para a abordagem da espiritualidade.

Alguns estudos também verificaram relações entre religiosidade e espiritualidade e resiliência em pacientes com doenças crônicas. Um estudo que avaliou 202 pacientes em hemodiálise verificou que resiliência correlacionou-se positivamente com religiosidade intrínseca, religiosidade organizacional, qualidade de vida e negativamente com depressão. ${ }^{37}$ Outro estudo envolvendo 82 pacientes de um centro de reabilitação de lesão medular encontrou associação positiva entre resiliência e suporte social e associação negativa entre resiliência e depressão. Não houve associação entre resiliência e espiritualidade. ${ }^{38}$ Estudo com 121 pacientes em cuidados paliativos (36,4\% destes em domicílio, e $85,1 \%$ por neoplasia) encontrou correlações significativas e positivas entre espiritualidade e resiliência, e negativas entre espiritualidade e depressão e ansiedade. ${ }^{39}$ Outro estudo com 105 pacientes internados em uma unidade de cuidados paliativos demonstrou correlações estatisticamente significativas e positivas entre espiritualidade e resiliência. ${ }^{40}$ 
No presente trabalho, houve correlação positiva entre resiliência e espiritualidade, achado que permite a hipótese de que a espiritualidade pode se comportar como um mecanismo promotor de resiliência. Entretanto, não foi encontrada associação entre resiliência e religiosidade intrínseca ou práticas religiosas privadas, fenômeno possivelmente melhor explicado pelos elevados índices observados para estas variáveis (79,6\% e 90,3\%, respectivamente), o que gera um efeito teto na correlação, aliado a um universo pequeno.

A escala de espiritualidade, por ter mais itens, permite que haja uma maior diferenciação entre as respostas dos sujeitos. Como a escala de espiritualidade ARES foi criada a partir da opinião de brasileiros e, dentre estes, muitos se utilizaram de elementos da religião para formar sua concepção de espiritualidade, ${ }^{19}$ pode-se pensar que suas questões se aproximam da ideia de religiosidade intrínseca.

Teoriza-se que espiritualidade e religiosidade funcionam como uma fonte de força e resiliência em situações de adversidade através de uma variedade de rotas. ${ }^{7}$ De acordo com a literatura, resiliência provavelmente é fruto de múltiplos preditores independentes, sendo cada um responsável por pequena parte da variação. ${ }^{32}$ Estudos sugerem que espiritualidade e religiosidade podem, na maioria das vezes, incentivar emoções positivas, fomentar suporte social espiritual e, de forma muito importante, promover a produção de sentido e ressignificação de experiências difíceis. ${ }^{7} \mathrm{Na}$ literatura, religiosidade intrínseca está habitualmente associada a estados mentais saudáveis. ${ }^{12}$

\section{Implicações para pesquisa e/ou prática profissional}

Este estudo contribui para o entendimento da relação entre a religiosidade e espiritualidade e a resiliência, que por sua vez está associada a estados mentais mais saudáveis, e demonstra o perfil religioso/ espiritual dos pacientes estudados que recebem atenção domiciliar.

Apesar de a dimensão espiritual fazer parte da integralidade do indivíduo e, ao lado do biológico, psicológico e social, estar no cerne do processo saúde-doença, ela é ainda muito pouco abordada na prática clínica. ${ }^{41}$ Diversos achados levam pesquisadores a concluir que a incorporação da abordagem da religiosidade e espiritualidade à prática clínica, avaliando o paciente como um todo, se faz cada vez mais essencial. ${ }^{42}$ Existe vasta literatura sobre como realizar uma anamnese espiritual, ${ }^{43}$ inclusive na perspectiva do Método Clínico Centrado na Pessoa. ${ }^{41}$ Identificar os fatores de sentido e significado de vida, validar práticas e crenças que Ihes façam bem e ressignificar dificuldades pode ter um potencial importante de suporte e de fomento da resiliência.

\section{CONCLUSÃO}

A população entrevistada possui alta taxa de religiosidade e espiritualidade. No presente estudo, resiliência apresentou associação significativa com espiritualidade e idade avançada. A associação de resiliência com espiritualidade se manteve estatisticamente significativa após controle para sintomas depressivos e suporte social. A maioria dos participantes nunca foi abordada por profissionais de saúde sobre religiosidade/espiritualidade em atendimentos, entretanto considera importante ser questionado sobre o tema no cenário de atendimento de saúde. Destaca-se o papel da APS e do MFC no cuidado integral dos pacientes em AD. 


\section{Contribuições dos autores}

Concepção e/ou delineamento do estudo: RBZ, BPM, EDCF, JBSS, RMB. Aquisição, análise ou interpretação dos dados: RBZ, BPM, EDCF, JBSS, RMB, DTS. Redação preliminar: RBZ, BPM, EDCF, JBSS, RMB, DTS. Revisão crítica da versão preliminar: RBZ, EDCF, BPM. Todos os autores aprovaram a versão final e concordaram com prestar contas sobre todos os aspectos do trabalho.

\section{REFERÊNCIAS}

1. Brasil. Ministério da Saúde. Secretaria de Atenção à Saúde. Departamento de Atenção Básica. Melhor em casa. A segurança do hospital no conforto do seu lar. Caderno de atenção domiciliar. Brasília (DF): Ministério da Saúde; 2012; [acesso 2018 Outubro 15]. Disponível em: http://189.28.128.100/dab/docs/publicacoes/geral/cad_vol1.pdf

2. Brasil. Ministério da Saúde. Envelhecimento e saúde da pessoa idosa. Caderno de atenção básica. Brasília (DF): Ministério da Saúde;2006.

3. United Nations (UN). Department of Economic and Social Affairs. World population prospects: the 2017 revision. Copenhagen: US; 2017. Disponível em: https://www.un.org/development/desa/publications/world-population-prospects-the-2017-revision.html

4. Livneh H, Antonak RF. Psychosocial adaptation to chronic illness and disability: a primer for counselors. J Couns Dev. 2005;83(1):12-20. DOI: https://doi.org/10.1002/j.1556-6678.2005.tb00575.x

5. Rutten BPF, Hammels C, Geschwind N, Menne-Lothmann C, Pishva E, Schruers K, et al. Resilience in mental health: linking psychological and neurobiological perspectives. Acta Psychiatr Scand. 2013;128(1):3-20. DOI: https://doi.org/10.1111/acps.12095

6. Patel V, Saxena S, Lund C, Thornicroft SG, Baingana F, Bolton P, et al. The Lancet Commission on global mental health and sustainable development. Lancet. 2018;392(10157):1553-98. DOI: https://doi.org/10.1016/S0140-6736(18)31612-X

7. Faigin CA, Pargament KI. Strengthened by the spirit: religion, spirituality, and resilience through adulthood and aging. Resilience Aging 2010:163-80. DOI: https://doi.org/10.1007/978-1-4419-0232-0_11

8. Stewart DE, Yuen T. A systematic review of resilience in the physically ill. Psychosomatics. 2011;52(3):199-209. DOI: https://doi.org/10.1016/j. psym.2011.01.036

9. Moreira-Almeida A, Pinsky I, Zaleski M, Laranjeira R. Envolvimento religioso e fatores sociodemográficos: resultados de um levantamento nacional no Brasil. Rev Psiquiatr Clin. 2010 Jan;37(1):12-5. DOI: https://doi.org/10.1590/S0101-60832010000100003

10. Shahid R, Zuettel I. Global Index of Religiosity and Atheism. Washington, DC: WIN-Gallup International; 2012; [acesso 2018 Setembro 9]. Disponível em: http://www.wingia.com/web/files/news/14/file/14.pdf

11. Koenig H, King D, Carson VB. Handbook of religion and health. Oxford: University Press; 2012.

12. Moreira-Almeida A, Lucchetti G. Panorama das pesquisas em ciência, saúde e espiritualidade. Ciênc Cult. 2016;68(1):54-7. DOI: https:// doi.org/10.21800/2317-66602016000100016

13. Li S, Stampfer MJ, Williams DR, VanderWeele TJ. Association of religious service attendance with mortality among women. JAMA Intern Med. 2016;176(6):777-85. DOI: https://doi.org/10.1001/jamainternmed.2016.1615

14. Brasil. Ministério da Saúde. Portaria №2.527, de 27 de outubro de 2011. Redefine a atenção domiciliar no âmbito do Sistema Único de Saúde (SUS). Brasília: Ministério da Saúde; 2011.

15. Lucchetti G, Lucchetti ALG, Peres MF, Leão FC, Almeida AM, Koenig HG. Validation of the duke religion index: DUREL (Portuguese Version). J Relig Health. 2012;51(2):579-86. DOI: https://doi.org/10.1007/s10943-010-9429-5

16. Martinez EZ, Alves AC, Carneiro AFTM, Jorge TM, Carvalho ACD, Zucoloto ML. Investigação das propriedades psicométricas do Duke Religious Index no âmbito da pesquisa em Saúde Coletiva. Cad Saúde Colet. 2014;22(4):419-27. DOI: https://doi.org/10.1590/1414$462 \times 201400040016$

17. Allport GW, Ross JM. Personal religious orientation and prejudice. J Pers Soc Psychol. 1967;5(4):432-43. DOI: https://doi.org/10.1037/ h0021212

18. Stroppa A, Moreira-Almeida A. Religiosity, mood symptoms, and quality of life in bipolar disorder. Bipolar Disord. 2013;15(4):385-93. DOI: https://doi.org/10.1111/bdi.12069 
19. Braghetta CC. Desenvolvimento e validação de um instrumento para avaliar espiritualidade: Escala de Atitudes Relacionadas à Espiritualidade (ARES) [dissertação]. São Paulo: Universidade de São Paulo - Faculdade de Medicina; 2017. DOI: https://doi. org/10.11606/D.5.2017.tde-05102017-112819

20. Damásio BF, Borsa JC, Silva JP. 14-item resilience scale (RS-14): psychometric properties of the Brazilian version. J Nurs Meas. 2011;19(3):131-45. DOI: https://doi.org/10.1891/1061-3749.19.3.131

21. Wagnild GM, Guinn PE. The Resilience Scale User's Guide. Worden, MT: Resilience Center; 2014.

22. Katz S, Ford AB, Moskowitz RW, Jackson BA, Jaffe MW. Studies of illness in the aged. The Index of ADL: a standardized measure of biological and psychosocial function. JAMA. 1963;185(12):94-9. DOI: https://doi.org/10.1001/jama.1963.03060120024016

23. Lino VTS, Pereira SRM, Camacho LAB, Ribeiro Filho ST, Buksman S. Adaptação transcultural da Escala de Independência em Atividades da Vida Diária (Escala de Katz). Cad Saúde Pública. 2008;24(1):103-12.

24. Wallace M, Shelkey M. Katz index of independence in activities of daily living (ADL). Am J Nurs. 2007;108(2):67-71.

25. Gaya CM. Estudo de validação de instrumentos de rastreamento para transtornos depressivos, abuso e dependência de álcool e tabaco [dissertação]. Ribeirão Preto (SP): Universidade de São Paulo - Faculdade de Medicina de Ribeirão Preto; 2009. DOI: https:// doi.org/10.11606/t.17.2011.tde-26092011-144558

26. Mosera A, Stuckb AE, Sillimanc RA, Ganzd PA, Clough-Gorra KM. The eight-item modified Medical Outcomes Study Social Support Survey: psychometric evaluation showed excellent performance. J Clin Epidemiol. 2012;65(10):1107-16. DOI: https://doi.org/10.1016/j. jclinepi.2012.04.007

27. Sherbourne CD, Stewart AL. The MOS social support survey. Soc Sci Med. 1991;32(6):705-14. DOI: https://doi.org/10.1016/02779536(91)90150-B

28. Griep RH, Chor D, Faerstein E, Werneck GL, Lopes CS. Validade de constructo de escala de apoio social do Medical Outcomes Study adaptada para o português no Estudo Pró-Saúde. Cad Saúde Pública. 2005;21(3):703-14. DOI: https://doi.org/10.1590/S0102$-311 X 2005000300004$

29. Associação Brasileira de Empresas de Pesquisa (ABEP). Critério Brasil 2015 e atualização da distribuição de classes para 2016 . São Paulo (SP): ABEP; 2016; [acesso 2018 Junho 12]. Disponível em: www.abep.org

30. Miller MD, Paradis CF, Houck PR, Mazumdar S, Stack JA, Rifai AH, et al. Rating chronic medical illness burden in geropsychiatric practice and research: application of the Cumulative Illness Rating Scale. Psychiatry Res. 1992;41(3):237-48. DOI: https://doi.org/10.1016/01651781(92)90005-n

31. Hudon C, Fortin M, Vanasse A. Cumulative Illness Rating Scale was a reliable and valid index in a family practice context. J Clin Epidemiol. 2005;58(6):603-8. DOI: https://doi.org/10.1016/j.jclinepi.2004.10.017

32. Mosqueiro BP, Rocha NS, Fleck MPA. Intrinsic religiosity, resilience, quality of life, and suicide risk in depressed inpatients. J Affect Disord. 2015;179:128-33. DOI: https://doi.org/10.1016/j.jad.2015.03.022

33. Lucchetti G, Lucchetti AG, Badan-Neto AM, Peres PT, Peres MFP, Moreira-Almeida A, et al. Religiousness affects mental health, pain and quality of life in older people in an outpatient rehabilitation setting. J Rehabil Med. 2011;43(4):316-22. DOI: https://doi. org/10.2340/16501977-0784

34. McCord G, Gilchrist VJ, Grossman SD, King BD, McCormick KF, Oprandi AM, et al. Discussing spirituality with patients: a rational and ethical approach. Ann Fam Med. 2004;2(4):356-61. DOI: https://doi.org/10.1370/afm.71

35. Ehman JW, Ott BB, Short TH, Ciampa RC, Hansen-Flaschen J. Do patients want physicians to inquire about their spiritual or religious beliefs if they become gravely ill?. Arch Intern Med. 1999;159(15):1803-6. DOI: https://doi.org/10.1001/archinte.159.15.1803

36. Oliveira JAC. Desafios do cuidado integral em saúde: a dimensão espiritual do médico se relaciona com sua prática na abordagem espiritual do paciente? [dissertação]. São Paulo (SP): Universidade de São Paulo - Faculdade de Medicina; 2018.

37. Medeiros CMMF, Arantes EP, Tajra RDP, Santiago HR, Carvalho AF, Libório AB. Resilience, religiosity and treatment adherence in hemodialysis patients: a prospective study. Psychol Health Med. 2017;22(5):570-7. DOI: https://doi.org/10.1080/13548506.2016.1191658

38. Bhattarai M, Maneewat K, Sae-Sia W. Psychosocial factors affecting resilience in Nepalese individuals with earthquake-related spinal cord injury: a cross-sectional study. BMC Psychiatry. 2018;18(1):60. DOI: https://doi.org/10.1186/s12888-018-1640-z

39. Tan JYS, Lim HA, Kuek NMY, Kua EH, Mahendran R. Caring for the caregiver while caring for the patient: exploring the dyadic relationship between patient spirituality and caregiver quality of life. Support Care Cancer. 2015;23(12):3403-6. DOI: https://doi.org/10.1007/ s00520-015-2920-5 
40. Redondo-Elvira T, Ibañez-del-Prado C, Barbas-Abad S. Espiritualmente resilientes. Relación entre espiritualidad y resiliencia en cuidados paliativos. Clín Salud. 2017;28(3):117-21. DOI: https://doi.org/10.1016/j.clysa.2017.09.001

41. Oliveira JAC, Anderson MIP, Lucchetti G, Ávila Pires EV, Gonçalves LM. Approaching spirituality using the patient-centered clinical method. J Relig Health. 2018 Jan;58:109-18. DOI: https://doi.org/10.1007/s10943-017-0534-6

42. Moreira-Almeida A, Koenig HG, Lucchetti G. Clinical implications of spirituality to mental health: review of evidence and practical guidelines. Rev Bras Psiquiatr. 2014;36(2):176-82. DOI: https://doi.org/10.1590/1516-4446-2013-1255

43. Lucchetti G, Granero AL, Bassi RM, Latorraca R, Nacif SAP. Espiritualidade na prática clínica: o que o clínico deve saber?. Rev Bras Clin Med. 2010;8(2):154-8. 